\title{
Analysis of Index Gases of Coal Spontaneous Combustion Using Fourier Transform Infrared Spectrometer
}

\author{
Xiaojun Tang, ${ }^{1}$ Yuntao Liang, ${ }^{2}$ Haozhe Dong, ${ }^{3}$ Yong Sun, ${ }^{2}$ and Haizhu Luo ${ }^{2}$ \\ ${ }^{1}$ State Key Laboratory of Electrical Insulation \& Power Equipment, Xi'an Jiaotong University, Xian 710049, China \\ ${ }^{2}$ State Key Laboratory of Coal Safety, Shenyang Branch of China Coal Research Institute, Shenyang 110016, China \\ ${ }^{3}$ Shenzhen Yetongda LtC, Shenzhen 518034, China
}

Correspondence should be addressed to Xiaojun Tang; xiaojun_tang@mail.xjtu.edu.cn

Received 14 July 2014; Accepted 18 September 2014; Published 20 October 2014

Academic Editor: Christoph Krafft

Copyright (C) 2014 Xiaojun Tang et al. This is an open access article distributed under the Creative Commons Attribution License, which permits unrestricted use, distribution, and reproduction in any medium, provided the original work is properly cited.

\begin{abstract}
Analysis of the index gases of coal for the prevention of spontaneous combustion is of great importance for the enhancement of coal mine safety. In this work, Fourier Transform Infrared Spectrometer (FTIRS) is presented to be used to analyze the index gases of coal in real time to monitor spontaneous combustion conditions. Both the instrument parameters and the analysis method are introduced at first by combining characteristics of the absorption spectra of the target analyte with the analysis requirements. Next, more than ten sets of the gas mixture containing ten components $\left(\mathrm{CH}_{4}, \mathrm{C}_{2} \mathrm{H}_{6}, \mathrm{C}_{3} \mathrm{H}_{8}\right.$, iso- $\mathrm{C}_{4} \mathrm{H}_{10}, n-\mathrm{C}_{4} \mathrm{H}_{10}, \mathrm{C}_{2} \mathrm{H}_{4}, \mathrm{C}_{3} \mathrm{H}_{6}, \mathrm{C}_{2} \mathrm{H}_{2}$, $\mathrm{CO}$, and $\mathrm{CO}_{2}$ ) are included and analyzed with a Spectrum Two FTIRS made by Perkin Elmer. The testing results show that the detection limit of most analytes is less than $2 \times 10^{-6}$. All the detection limits meet the monitoring requirements of coal spontaneous combustion in China, which means that FTIRS may be an ideal instrument and the analysis method used in this paper is sufficient for spontaneous combustion gas monitoring on-line and even in situ, since FTIRS has many advantages such as fast analysis, being maintenance-free, and good safety.
\end{abstract}

\section{Introduction}

Spontaneous combustion of coal is one of the most common causes of coal mine accidents. Quantitative analysis of index gases of coal spontaneous combustion is an important measure for monitoring the state of spontaneous combustion of coal [1-3]. It is of more importance for the safe production of coal since many mine disasters related to coal spontaneous combustion have ever happened. The index gases of coal spontaneous combustion are sometimes referred to as indicator gases or mark gases. They can be divided into two groups, majority (basic) and minority (additional) gases. The majority gases include $\mathrm{CO}, \mathrm{CH}_{4}, \mathrm{CO}_{2}$, and $\mathrm{O}_{2}$ while the minority gases include $\mathrm{C}_{2} \mathrm{H}_{6}, \mathrm{C}_{3} \mathrm{H}_{8}$, iso- $\mathrm{C}_{4} \mathrm{H}_{10}, n-\mathrm{C}_{4} \mathrm{H}_{10}, \mathrm{C}_{2} \mathrm{H}_{4}, \mathrm{C}_{3} \mathrm{H}_{6}$, $\mathrm{C}_{2} \mathrm{H}_{2}$, and $\mathrm{H}_{2}$ [1]. Although, only some components of the above are used for coal spontaneous combustion forecasting [4], in recent years, both majority gases and minority gases are required in most countries. For instance, in Poland, the assessment of minority gases is recommended [5]. In the
Czech Republic, the State Mining Authority has mandated the monitoring of both the majority and minority of gases in their entirety under specific conditions in underground mines of the Ostrava-Karvina Coal field (OKC) [6]. In China, $\mathrm{CH}_{4}$, $\mathrm{C}_{2} \mathrm{H}_{6}, \mathrm{C}_{3} \mathrm{H}_{8}$, iso- $\mathrm{C}_{4} \mathrm{H}_{10}, n-\mathrm{C}_{4} \mathrm{H}_{10}, \mathrm{C}_{2} \mathrm{H}_{4}, \mathrm{C}_{3} \mathrm{H}_{6}, \mathrm{C}_{2} \mathrm{H}_{2}, \mathrm{CO}$, and $\mathrm{CO}_{2}$ are required to be monitored [7].

Nowadays, many researchers focused on the state analysis of spontaneous combustion of coal and the interpretation of spontaneous temperature, and much progresses has been realized $[2,8-10]$. However, it is regretful that the interpretation in question encounters significant inaccuracies [1]. The inaccuracies are caused by many reasons. Asides from the fact that the exact mechanism of the reaction of oxygen with coal is not completely understood as the chemical nature of coal is not yet fully established [2], the detection technology itself may be one of the important factors. For early detection of spontaneous combustion of coal, the studies have shown that some index gases such as ethylene start to occur when 
spontaneous combustion reaches a characteristic temperature. However, if the state of spontaneous combustion of coal is forecasted online with beam tube monitoring system or offline with gas chromatograph (GC) in laboratory, it is difficult to detect these gases until the state of the spontaneous combustion has developed and is past its early stage. This is primarily due to dilution of ventilation air and high detection limits of the instruments used for gas analysis at coal mines [3]. Furthermore, an optimum opportunity for mine operators to control the hazard may be missed in the field of fire control of the coal mine. In fact, online and in situ monitoring is of great importance for safety because it can obtain real-time data and eliminate potential contamination and other errors that can be introduced during sample collection, transportation, and storage. Therefore, it is necessary to detect these index gases online and in situ for the early detection of spontaneous combustion of coal.

The most widely used techniques for multicomponent gas analysis are GC and optical absorption $[2,6,7,11]$. Although it is said that there are many advantages, such as high selectivity, low measurement limit, wide dynamic range, and short response time (typically below $10 \mathrm{~s}$ ) with using photoacoustic for this purpose, this technique is not yet widely accepted [11]. In fact, FT-IR has been widely used in many fields for gas analysis [12, 13], including fire gases analysis [14], because many compounds have the potential to produce an infrared absorption spectrum through the existence of vibrating dipoles in their molecular structure. Additionally, FT-IR has many advantages compared with GC, such as having no consumables and maintenance-free, fast analysis, intrinsical safety, low cost, and working online and even in situ. The primary factor prohibiting its application is the overlapping spectra when there are several components with the same function group. On the other hand, if an advanced algorithm is developed and applied, it is not a problem since there are not two completely overlapping spectra $[13,15]$. We have ever tried to analyze light alkane gas mixture with FTIRS, and high accuracies have been obtained [15]. FT-IR results are very encouraging since it provides an extremely robust, accurate, real-time analysis of index gas that will greatly enhance fire safety.

In this paper, both the spectrometer parameters and analysis approach have been introduced at first. For the analysis approach, baseline correction, feature selection, and analysis model building have been involved. Next, ten analytes required for coal spontaneous combustion forecast in China have been analyzed to determine if the approach used in this work meets the requirements of index gases of coal spontaneous combustion in China. The analyzing results show that detection limits of all components of the analytes are less than $2 \times 10^{-6}$. In particular, the testing errors of the key gases $\left(\mathrm{C}_{2} \mathrm{H}_{4}\right.$ and $\left.\mathrm{C}_{2} \mathrm{H}_{2}\right)$ are less than $1 \times$ $10^{-6}$ when their practical concentrations are less than $10 \times$ $10^{-6}$. Because FTIRS can be utilized in coal mines, the work of this paper may show the great advantages for the index gases monitoring of coal spontaneous combustion online and even in situ. Additionally, some novel portable spectrometers, such as Spectrum Two IR Spectrometer from Perkin Elmer, have been developed and marketed. This makes it possible to patrol the safety of coal mines with such an instrument by patrolmen.

\section{Experiments}

2.1. Instrument Parameters and Sample Preparation. For this work, Spectrum Two IR Spectrometers, one new type of FTIRS developed in the year of 2011 and made in 2013 by Perkin Elmer, is used. The optical path is $10 \mathrm{~cm}$. The spectral resolution is set as $1 \mathrm{~cm}^{-1}$. The scanning time is set to 16 . During the process of scanning absorption spectra of the samples, Norton-Beer medium function is selected as the apodization function since it can lead to the best fitting adherence to Beer's law for the spectra measured at moderate resolution. Because the gas concentration is affected by temperature and pressure variation, they must be held constant. In this work, ambient temperature of instrument and gas cylinders is kept at a constant $20^{\circ} \mathrm{C}$ with an air-conditioner. The pressure of the analyte during the scanning is also kept constant through the following procedure.

(1) Between two outlets of the gas cell, one is connected with gas cylinder while the other is connected with a long tube to emit the sample gas. The length of the tube is $10 \mathrm{~m}$; and the inside diameter is $3 \mathrm{~mm}$.

(2) Nitrogen is injected into the gas cell to purge it. The flow is controlled at $1 \mathrm{~L} / \mathrm{min}$ with flow controller. About 60 minutes, turn off the cylinder; wait for 30 seconds and turn off the switch connected with the tube to keep the pressure in the gas cell equal to atmospheric pressure; and then scan the background spectrum.

(3) Replace the nitrogen cylinder with one of the sample cylinders; turn on the switch and then the sample cylinder is injected with the sample gas into gas cell. About 30 minutes later, turn off the cylinder; scan sample spectrum; run the analysis software, and one sample has been tested.

(4) Repeat step (3) to get the spectrum of each sample until all of the sample spectra have been collected.

For every component of the gas, both the single component of the sample and the mixture sample are prepared. Totally, 120 samples have been prepared for calibrating the instrument and 15 sets of the samples for testing them. The concentration range of every component of the analyte is shown in Table 1. In fact, there is no definite concentration range of the index gas in the performance requirements of the instrument in China. Only the upper detection limits of $\mathrm{CO}$, $\mathrm{CH}_{4}$, and $\mathrm{CO}_{2}$ are required to be greater than $5000 \times 10^{-6}$ [7]. The concentration range of every component of the analyte in Table 1 is determined according to [7]. In a coal mine, if the concentration of any one component of the analyte reaches its upper limit in Table 1, there may be serious potential of hazard coal mine condition. And some measures must be taken to control the coming hazard. 
TABLE 1: Concentration range of every component of index gas.

\begin{tabular}{lcc}
\hline Item & Gas & Range \\
\hline 1 & $\mathrm{CH}_{4}$ & $0 \sim 200000 \times 10^{-6}$ \\
2 & $\mathrm{C}_{2} \mathrm{H}_{6}$ & $0 \sim 10000 \times 10^{-6}$ \\
3 & $\mathrm{C}_{3} \mathrm{H}_{8}$ & $0 \sim 5000 \times 10^{-6}$ \\
4 & $i \mathrm{C}_{4} \mathrm{H}_{10}$ & $0 \sim 5000 \times 10^{-6}$ \\
5 & $n \mathrm{C}_{4} \mathrm{H}_{10}$ & $0 \sim 5000 \times 10^{-6}$ \\
6 & $\mathrm{C}_{2} \mathrm{H}_{4}$ & $0 \sim 5000 \times 10^{-6}$ \\
7 & $\mathrm{C}_{3} \mathrm{H}_{6}$ & $0 \sim 3000 \times 10^{-6}$ \\
8 & $\mathrm{C}_{2} \mathrm{H}_{2}$ & $0 \sim 3000 \times 10^{-6}$ \\
9 & $\mathrm{CO}_{1}$ & $0 \sim 10000 \times 10^{-6}$ \\
10 & $\mathrm{CO}_{2}$ & $0 \sim 200000 \times 10^{-6}$ \\
\hline
\end{tabular}

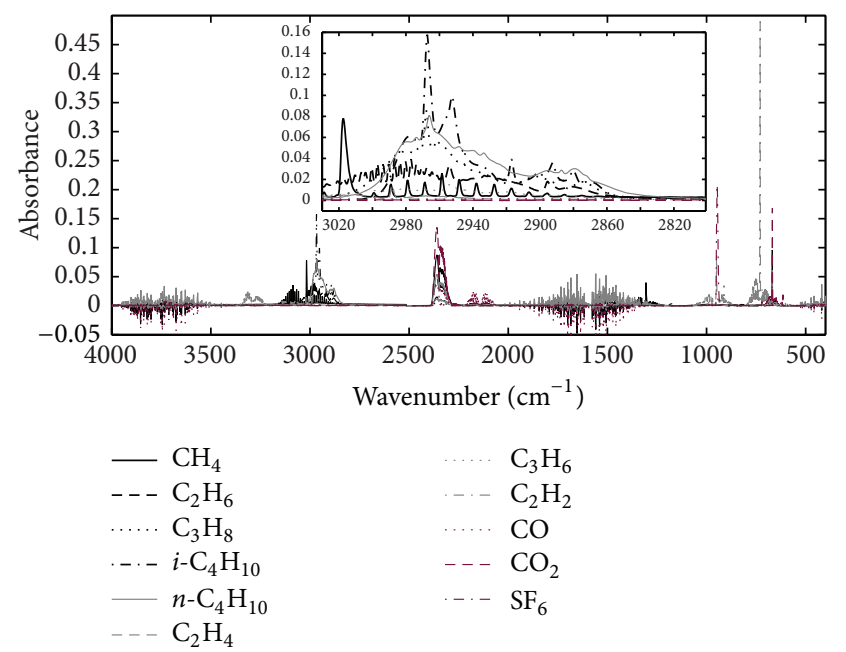

FIGURE 1: Absorption spectra of index gases of spontaneous combustion of coal.

2.2. Analysis Approach of Analyte. The absorption spectrum of every component of the analyte, whose concentration is $1000 \times 10^{-6}$, is shown in Figure 1. Please note that the balance gas of all the samples shown in Figure 1 is chosen as air, but not nitrogen, to show if water vapor content contaminates the analysis results of the index gases since there is always vapor in air. There are two absorption regions of vapor in middle infrared spectrum. One ranges from $1350 \mathrm{~cm}^{-1}$ to $2000 \mathrm{~cm}^{-1}$; the other ranges from $3500 \mathrm{~cm}^{-1}$ to $3900 \mathrm{~cm}^{-1}$. From Figure 1, it can be determined that, for every component of the analyte, there exists at least one absorption peak that does not overlap with the absorption regions of vapor. Therefore, it is easy to avoid the interference brought from vapor to the analysis result of every component of index gas if these absorption peaks are used to analyze the index gases.

During the process of the gas analysis online with FTIRS, at least two procedures are needed. One is spectrum preprocess to reduce the effects of the interferents and the instrument working conditions to the gas analysis results. The other is the analysis model calculation. Therefore, the spectrum preprocess approach must be selected according to practical applications, and the analysis model must be built at first. Then, the software is edited to implement these algorithms. In this subsection, the interferents needed to be determined are not in the scope of this paper, and only the spectrum baseline has been taken into account for the spectrum preprocess. For the analysis model, feature extraction is used to improve the selection of the inputs of the analysis model, and the partial least square (PLS) is used to build the analysis model of every component of the index gas.

2.2.1. Baseline Correction. After operating for a long time, even during the process of spectrum scanning, the baseline of spectrum may drift and even distort due to temperature change, the tilt of the reflect mirror, and the shift of detector, which may borrow error to the analysis result [13]. Therefore, the baseline must be corrected before the spectrum is used to quantitatively analyze the gas concentration.

For the spectrum baseline correction, several approaches have ever been proposed and applied, including derivative method [16], polynomial fitting [16], wavelet transform method [17], robust local regression [18], Asymmetric Least Squares (ALS) [19], and adaptive iteratively reweighted Penalized Least Squares (airPLS) [20]. Among these approaches, polynomial fitting is the most common used and comes in many different variants. From Figure 1, it can be found that there are several wavenumber zones where the absorbances of all the analytes are close to 0 , which can be taken as fitting points for correcting spectrum baselines with the polynomial fitting. The polynomial fitting may be a good choice to correct the baseline of the original spectrum since it has less calculation relative to others. In this work, five wavenumber zones, $560 \mathrm{~cm}^{-1}, 1120 \mathrm{~cm}^{-1}, 2000 \mathrm{~cm}^{-1}, 2500 \mathrm{~cm}^{-1}$, and $3400 \mathrm{~cm}^{-1}$ are taken as the center spectral line of these five zones, respectively, and are taken as fitting points for the baseline correction.

During the process of baseline correction, for every fitting point presented above, ten continuous absorbances are averaged at first. Next, a third-order polynomial curve is fitted with these averages and is taken as the spectrum baseline. Finally, it is subtracted from the original spectrum, and a corrected spectrum is obtained. In fact, the spectra shown in Figure 1 comprise only the baseline correction. For the original spectrum, there may be slight baseline drift. For instance, for the original spectrum of methane, as shown in Figure 2, the baseline decreases from 0 to 0.03 as wavenumber increases from 400 to $4000 \mathrm{~cm}^{-1}$. The baseline of the corrected spectrum is almost a perfect line whose value is 0 , except for the absorption region of water and $\mathrm{CO}_{2}$.

2.2.2. Analysis Model. Feature selection is often used to improve the selectivity of the inputs of the analysis model and thereby decrease the complexity of the analysis model. Nowadays, many approaches of feature selection have been reported. Forward selection (FS), PCA, stepwise regression (SWR), ridge regression (RR), genetic algorithm (GA), TR, and so on are included [21].

From Figure 1, it can be found that the spectra of some analytes have good selectivity while those of the others overlap with each other. For instance, the spectrum of propane 


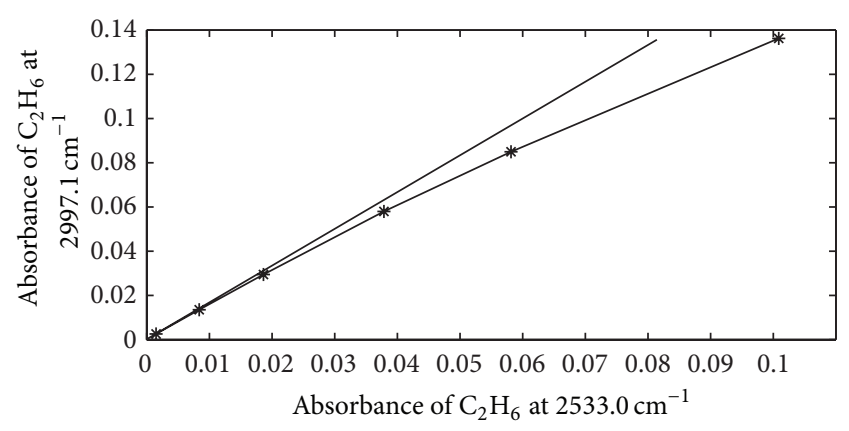

Figure 2: Absorbance of $\mathrm{C}_{2} \mathrm{H}_{6}$ at $2933.0 \mathrm{~cm}^{-1}$ versus that at $2997.1 \mathrm{~cm}^{-1}$.

overlaps that of $n$-butane while the absorption peak of $\mathrm{C}_{2} \mathrm{H}_{2}$ protrudes very far. The feature selection is necessary to extract inputs for the analysis models of propane and $n$-butane.

In this work, both forward selection and Tikhonov Regularization (TR) are used to extract the feature for every component of the gas with these corrected spectra to improve their selectivity. The latter is used for ethane, propane, iso-butane, and $n$-butane because their absorption spectra overlap with each other severely while the former is used for the others.

For the gas analysis with FTIRS, many calibration approaches have been reported. The principal PCA, TR, multivariate curve resolution (MCR), PLS, the neural network (NN), support vector regression (SVR), and so on [15] are included. Among these approaches, PLS may be one of the most widely used. TR can be used to perform the feature selection and build a linear model for the gas analysis. Because TR minimizes both model error and coefficients, it has been used widely in recent years. Both NN and SVR are usually used in such cases where nonlinearity is relatively extreme and spectra overlapping is severe. NN needs a large number of calibration samples while SVR is suitable for small sample sets.

According to [13], when the practical absorbance is less than 0.7 , it is almost linear to the gas concentration if the spectral resolution is less than the full width at half height of the absorption peak. From Figure 1, it can be found that there are many narrow discrete absorption peaks in the spectrum of $\mathrm{CH}_{4}$. Between $2950 \mathrm{~cm}^{-1}$ and $3050 \mathrm{~cm}^{-1}$, there are also some narrow absorption peaks in the spectrum of $\mathrm{C}_{2} \mathrm{H}_{6}$. The full width at half height of these absorption peaks is so close to spectral resolution that some absorbances may be slightly nonlinear to the gas concentration.

In this work, for the convenience of discussion, $A_{G, x}$ is used to denote the absorbance of analyte $G$ at $x \mathrm{~cm}^{-1}$ and $S_{G, x}$ denotes the corresponding sensitivity. For ins-tance, $A_{\mathrm{C}_{2} \mathrm{H}_{6}, 2933.0}$ denotes the absorbance of $\mathrm{C}_{2} \mathrm{H}_{6}$ at $2933.0 \mathrm{~cm}^{-1}$. In order to exemplify this point of view, the relation $A_{\mathrm{C}_{2} \mathrm{H}_{6}, 2933.0}$ versus $A_{\mathrm{C}_{2} \mathrm{H}_{6}, 2997.1}$ as concentration of $\mathrm{C}_{2} \mathrm{H}_{6}$ increase has been shown in Figure 3. Normally, if two absorbances are linear to the sample concentration completely, one of them is also linear to the other. But from Figure 3, it can be found that $A_{\mathrm{C}_{2} \mathrm{H}_{6}, 2997.1}$ increases more

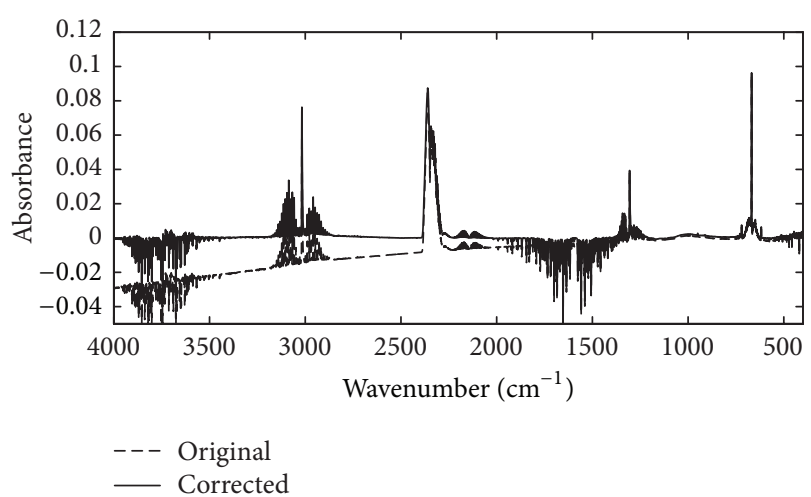

FIGURE 3: Spectra of methane before and after baseline correction has been done.

slowly than $A_{\mathrm{C}_{2} \mathrm{H}_{6}, 2933.0}$ as the concentration increases. So there is at least one absorbance that is nonlinear to the concentration of $\mathrm{C}_{2} \mathrm{H}_{6}$. In fact, it can be determined that the absorbance that is nonlinear to the concentration of $\mathrm{C}_{2} \mathrm{H}_{6}$ is $A_{\mathrm{C}_{2} \mathrm{H}_{6}, 2997.1}$, but not $A_{\mathrm{C}_{2} \mathrm{H}_{6}, 2933.0}$, since $A_{\mathrm{C}_{2} \mathrm{H}_{6}, 2997.1}$ is one of the absorbances in a narrow absorption peak, and $A_{\mathrm{C}_{2} \mathrm{H}_{6}, 2933.0}$ is not. Therefore, in this work, some absorbances are linear to the concentration of the analytes while the others have slight nonlinearity. Consequently, PLS may be more suitable for building the analysis model for every component of the analyte among above approaches.

For the analysis model, the inputs are one or several of the extracted features determined according to its selectivity, and the output is the concentration of one component of the analyte. For some analytes such as $\mathrm{CO}$ and $\mathrm{CO}_{2}$, their absorption spectra have good selectivity and there is only one feature for their analysis model. For the others, such as propane and $n$-butane, their features have slight crosssensitivity, and more than two features are needed. The model is a polynomial for some analytes.

During the process of analyzing the index gases of coal spontaneous combustion on-line, the spectrum is obtained with the spectrometer periodically and corrected at first and then used to extract the features. Finally, the analysis results are determined by calculating these analysis models.

\section{Testing Result and Analysis}

For the gas analysis online, the testing limit and the accuracy are two important indexes. In order to test the performance of our instrument calibrated with the sample prepared above, an additional 15 standard samples are analyzed.

3.1. Detection Limit. According to the requirement in China, the detection limit has not been given, but it has been given that the sensitivity of every component of index gases must be higher than $2 \times 10^{-6}$ [7]. Additionally, precise requirements of the index gases have also been given as Table 2. In Table 2, both the repeatability and the reproducibility have been given. Since the repeatability error is $3 \times 10^{-6}$ when the concentration ranges from 0 to $50 \times 10^{-6}$, to a certain degree, the detection limit can be taken as $3 \times 10^{-6}$. Certainly, if a 
TABLE 2: Precision requirement of index gases in China.

\begin{tabular}{lcc}
\hline Concentration range & Repeatability & Reproducibility \\
\hline $0 \sim 50 \times 10^{-6}$ & $3 \times 10^{-6}$ & $7 \times 10^{-6}$ \\
$50 \sim 500 \times 10^{-6}$ & $10 \times 10^{-6}$ & $15 \times 10^{-6}$ \\
$500 \sim 10000 \times 10^{-6}$ & $40 \times 10^{-6}$ & $70 \times 10^{-6}$ \\
\hline
\end{tabular}

TABLE 3: Mean square root of the analyte and maximal analysis error in case of background spectra.

\begin{tabular}{lccc}
\hline Item & $\mathrm{Gas}$ & Mean square root & Maximal error \\
\hline 1 & $\mathrm{CH}_{4}$ & $0.45 \times 10^{-6}$ & $0.88 \times 10^{-6}$ \\
2 & $\mathrm{C}_{2} \mathrm{H}_{6}$ & $0.28 \times 10^{-6}$ & $1.14 \times 10^{-6}$ \\
3 & $\mathrm{C}_{3} \mathrm{H}_{8}$ & $1.0 \times 10^{-6}$ & $1.9 \times 10^{-6}$ \\
4 & $i s o-\mathrm{C}_{4} \mathrm{H}_{10}$ & $0.4 \times 10^{-6}$ & $0.80 \times 10^{-6}$ \\
5 & $n-\mathrm{C}_{4} \mathrm{H}_{10}$ & $1.0 \times 10^{-6}$ & $1.8 \times 10^{-6}$ \\
6 & $\mathrm{C}_{2} \mathrm{H}_{4}$ & $0.48 \times 10^{-6}$ & $1.1 \times 10^{-6}$ \\
7 & $\mathrm{C}_{3} \mathrm{H}_{6}$ & $1.0 \times 10^{-6}$ & $1.9 \times 10^{-6}$ \\
8 & $\mathrm{C}_{2} \mathrm{H}_{2}$ & $0.16 \times 10^{-6}$ & $0.31 \times 10^{-6}$ \\
9 & $\mathrm{CO}_{10}$ & $0.61 \times 10^{-6}$ & $1.9 \times 10^{-6}$ \\
10 & $\mathrm{CO}_{2}$ & $0.3 \times 10^{-6}$ & $1.0 \times 10^{-6}$ \\
\hline
\end{tabular}

strict requirement is needed, the detection limit can be taken as $2 \times 10^{-6}$.

For the detection limit of every component of the index gases, it can be tested directly with the background spectra. In this work, 30 background spectra have been scanned continuously and analyzed with the software developed in this work. Next, the standard deviation (root-mean-square error: RMS error) and maximal error of every component have been calculated. As listed in Table 3, it can be found that the maximal error of every component of the index gases is less than $2 \times 10^{-6}$. But on the other hand, the maximal testing errors of $\mathrm{C}_{3} \mathrm{H}_{8}, n-\mathrm{C}_{4} \mathrm{H}_{10}, \mathrm{C}_{3} \mathrm{H}_{6}$, and $\mathrm{CO}$ are close to $2 \times 10^{-6}$ and the margin is very low if $2 \times 10^{-6}$ is taken as the detection limit. Besides, because of overlapping absorption spectra, $\mathrm{C}_{3} \mathrm{H}_{8}$ and $n \mathrm{C}_{4} \mathrm{H}_{10}$ may bring effect to each other although their selectivity has been improved through features extraction. In other words, the concentration of one of them may change slightly as that of the other. When the concentration of one component is 0 , that of the other is high and its detection limit may exceed $2 \times 10^{-6}$ and even $3 \times$ $10^{-6}$ because of serious overlapping of the absorption spectra. Certainly, since the concentrations of all the hydrocarbon gases and $\mathrm{CO}$ increase as that of the temperature of coal spontaneous combustion [1], the concentration of $n$-butane must not be 0 when the concentration of propane is high, especially, the concentrations of these two components are close to each other sometimes. However, this case will not happen. Therefore, it is acceptable if the maximal testing error is taken as detection limit list in Table 3.

3.2. Accuracy. The analyzed results of the fifteen testing samples are shown in Table 4. In Table 4, Exp means the practical concentrations while Anal means the analyzed results of every component of the gas.
From Table 4, the following can be shown.

(1) For every component of the analyte in the testing samples, its practical concentrations cover its range.

(2) When the practical concentration of every component is less than $50 \times 10^{-6}$, the analysis error is less than $7 \times 10^{-6}$. In other words, all the testing results fit the requirement of reproducibility shown in Table 2.

(3) When the practical concentration is higher than $50 \times$ $10^{-6}$, for some components, the maximum analysis error exceeds the requirement of reproducibility shown in Table 2 . For instance, among the 4th set of testing results, the testing result of $n$-butane is $82.2 \times 10^{-6}$ while its practical concentration is $100 \times 10^{-6}$. The analysis error is $17.8 \times 10^{-6}$ and higher than $15 \times 10^{-6}$, the corresponding requirement of reproducibility. For these testing results, their background colors have been denoted with grey. It can be shown that there are 10 such testing results whose errors exceed their corresponding reproducibility. Additionally, according to Table 2, the precision requirement of the index gases is only limited by the concentration range from 0 to $1 \% / 10000 \times$ $10^{-6}$. In fact, the concentration range of methane and carbon dioxide covers 0 to $20 \%$. When the concentration of methane or carbon dioxide is further increased to higher than $1 \%$, the analysis error is higher than $70 \times 10^{-6}$, the maximum admissible reproducibility error, in many cases. For these analysis results, they have been denoted with an italic font. From Table 4, it can be found there are 10 such analysis results. As a result, according to Table 2, the qualified ratio of analysis results in the stated concentration range is about $(10 \times 15-10-10) \times 100 \% /(10 \times 15-10) \approx 92.8 \%$. But on the other hand, relative analysis error is often used to evaluate the performance of the gas analysis instrument when the gas concentration reaches a certain value [14]. In Table 5, both the maximum absolute analysis error and the maximum relative error of every component of the analyte have been listed when the concentration is higher than $50 \times 10^{-6}$ but still in the concentration range listed in Table 1. From Table 5, it can be found that the maximum relative error is $5.8 \%$. For the gas analysis online, it is an acceptable result, especially when there are several components of the gases whose absorption spectra overlap with each other extensively [22]. In fact, according to the coal temperature experimental results, the concentration of the same gas emitted by the different coal samples at same temperature differs from each other largely. For instance, according to [1], the desorption of $n$-butane of different coal sample ranges from 5 to $26 \mathrm{~mL} / \mathrm{t}$.min when the temperature is $160^{\circ} \mathrm{C}$. If their average value is taken as their practical value, the maximum relative error increases to $68.7 \%$ which is much greater than $5.8 \%$. And the effect brought from such relative analysis error of the index gases to forecast the results can almost be neglected relative to that brought from the coal temperature experiment results if these index gases are used to forecast coal spontaneous combustion.

(4) In the 9th set of the testing results, although the practical concentration of methane and that of ethane exceed their respective concentration ranges, such calibration samples have not been prepared and the testing results are also 
TABLE 4: Testing results.

\begin{tabular}{|c|c|c|c|c|c|c|c|c|c|c|}
\hline \multirow{2}{*}{ Item } & \multicolumn{10}{|c|}{ Concentration of index gas $/ \varphi(\cdot) \times 10^{-6}$} \\
\hline & $\mathrm{CH}_{4}$ & $\mathrm{C}_{2} \mathrm{H}_{6}$ & $\mathrm{C}_{3} \mathrm{H}_{8}$ & $i \mathrm{C}_{4} \mathrm{H}_{10}$ & $n \mathrm{C}_{4} \mathrm{H}_{10}$ & $\mathrm{C}_{2} \mathrm{H}_{4}$ & $\mathrm{C}_{3} \mathrm{H}_{6}$ & $\mathrm{C}_{2} \mathrm{H}_{2}$ & $\mathrm{CO}$ & $\mathrm{CO}_{2}$ \\
\hline \multicolumn{11}{|l|}{1} \\
\hline Exp & 976 & 19.8 & 0 & 0 & 0 & 0 & 0 & 0 & 0 & 760 \\
\hline Anal & 986 & 18.7 & 0.0 & 0.7 & 0.0 & 0.5 & 1.0 & 0.2 & 1.8 & 764 \\
\hline \multicolumn{11}{|l|}{2} \\
\hline Exp & 99972 & 998 & 199 & 0 & 0 & 0 & 0 & 0 & 0 & 0 \\
\hline Anal & 98823 & 981 & 202 & 0.3 & 3.5 & 0.2 & 0.4 & 0.1 & 1.5 & 0.0 \\
\hline \multicolumn{11}{|l|}{3} \\
\hline Exp & 99819 & 498 & 199 & 50.0 & 50.0 & 50.0 & 50.0 & 50.0 & 10.0 & 230 \\
\hline Anal & 100000 & 487 & 212 & 47.5 & 56.1 & 45.3 & 52.2 & 48.6 & 9.0 & 231 \\
\hline \multicolumn{11}{|l|}{4} \\
\hline Exp & 19988 & 498 & 199 & 100 & 100 & 498 & 200 & 50.4 & 15.0 & 1010 \\
\hline Anal & 19880 & 486 & 214 & 95.1 & 82.2 & 467 & 205 & 47.4 & 15.3 & 1022 \\
\hline \multicolumn{11}{|l|}{5} \\
\hline Exp & 199790 & 9996 & 2987 & 2993 & 2497 & 2989 & 998 & 999 & 600 & 10020 \\
\hline Anal & 201060 & 10221 & 3159 & 3053 & 2565 & 2920 & 1051 & 1007 & 611 & 10172 \\
\hline \multicolumn{11}{|l|}{6} \\
\hline Exp & 10001 & 497 & 100 & 50.0 & 30.0 & 0 & 0 & 0 & 20 & 490 \\
\hline Anal & 9970 & 485 & 92.3 & 52.4 & 28.3 & 0.9 & 0.0 & 0.4 & 22 & 494 \\
\hline \multicolumn{11}{|l|}{7} \\
\hline Exp & 10011 & 501 & 100 & 50.0 & 100 & 19.8 & 9.7 & 0 & 200 & 2520 \\
\hline Anal & 10309 & 499 & 86.9 & 53.5 & 106 & 17.4 & 10.8 & 0.3 & 198 & 2503 \\
\hline \multicolumn{11}{|l|}{8} \\
\hline Exp & 99892 & 2021 & 1001 & 500 & 602 & 501 & 200 & 100 & 300 & 2300 \\
\hline Anal & 99619 & 2126 & 1012 & 494 & 590 & 510 & 202 & 97 & 309 & 2327 \\
\hline \multicolumn{11}{|l|}{9} \\
\hline Exp & 501020 & 20012 & 4987 & 4993 & 5017 & 4989 & 2998 & 2998 & 5002 & 200020 \\
\hline Anal & 511060 & 20722 & 5059 & 5053 & 5065 & 4962 & 3051 & 3016 & 4911 & 201703 \\
\hline \multicolumn{11}{|l|}{10} \\
\hline Exp & 45004 & 2000 & 1000 & 500 & 400 & 20.1 & 10.1 & 10.2 & 10.2 & 2200 \\
\hline Anal & 44636 & 2030 & 1051 & 489 & 405 & 20.4 & 10.0 & 9.7 & 9.2 & 2219 \\
\hline \multicolumn{11}{|l|}{11} \\
\hline Exp & 198972 & 978 & 255 & 302 & 200 & 49.8 & 40.0 & 19.8 & 199 & 700 \\
\hline Anal & 196650 & 960 & 264 & 307 & 201 & 50.7 & 40.2 & 20.1 & 201 & 690 \\
\hline \multicolumn{11}{|l|}{12} \\
\hline Exp & 198 & 18.1 & 15.0 & 10.0 & 0 & 0 & 0 & 0 & 0 & 504 \\
\hline Anal & 201 & 16.3 & 16.1 & 10.8 & 0.9 & 0 & 0.2 & 0.0 & 0.5 & 500 \\
\hline \multicolumn{11}{|l|}{13} \\
\hline Exp & 502 & 10.1 & 5.0 & 0 & 0 & 0 & 0 & 0 & 0 & 20.2 \\
\hline Anal & 501 & 9.2 & 4.2 & 0.1 & 1.2 & 0.2 & 0.1 & 0.2 & 0.9 & 18.6 \\
\hline \multicolumn{11}{|l|}{14} \\
\hline Exp & 39995 & 1987 & 100 & 40.0 & 30.0 & 20.0 & 19.9 & 0 & 400 & 998 \\
\hline Anal & 39534 & 1956 & 96.1 & 39.1 & 27.1 & 18.1 & 18.7 & 0.3 & 387 & 986 \\
\hline \multicolumn{11}{|l|}{15} \\
\hline Exp & 1989 & 98.2 & 19.0 & 10.0 & 0 & 10.0 & 0 & 5 & 0 & 0 \\
\hline Anal & 1925 & 96.1 & 17.1 & 10.2 & 1.1 & 9.8 & 0.0 & 5.5 & 0.1 & 0.0 \\
\hline
\end{tabular}


TABLE 5: The maximum absolute error and the maximum relative error of every component analysis result.

\begin{tabular}{|c|c|c|c|c|c|c|c|c|c|c|}
\hline & $\mathrm{CH}_{4}$ & $\mathrm{C}_{2} \mathrm{H}_{6}$ & $\mathrm{C}_{3} \mathrm{H}_{8}$ & iso- $\mathrm{C}_{4} \mathrm{H}_{10}$ & $n-\mathrm{C}_{4} \mathrm{H}_{10}$ & $\mathrm{C}_{2} \mathrm{H}_{4}$ & $\mathrm{C}_{3} \mathrm{H}_{6}$ & $\mathrm{C}_{2} \mathrm{H}_{2}$ & $\mathrm{CO}$ & $\mathrm{CO}_{2}$ \\
\hline Maximum error $/ \varphi(\cdot) \times 10^{-6}$ & 2320 & 225 & 172 & 60 & 68 & 59 & 53 & 8 & 11 & 152 \\
\hline Sample number & 11 & 5 & 5 & 5 & 5 & 5 & 5 & 5 & 5 & 5 \\
\hline Maximum relative error & $3.2 \%$ & $5.2 \%$ & $5.8 \%$ & $2.2 \%$ & $2.7 \%$ & $2.0 \%$ & 5.3 & $0.8 \%$ & $1.8 \%$ & $1.7 \%$ \\
\hline Sample number & 15 & 8 & 5 & 10 & 5 & 5 & 5 & 8 & 5 & 5 \\
\hline
\end{tabular}

acceptable since their relative errors are only $2.0 \%$ and $3.5 \%$, respectively. Such accurate analysis results are attributable to good monotonicity of absorbance to the gas concentration and there are some absorbances with good selection for methane and ethane. This makes it possible to get good prediction accuracy from the analysis models of index gases.

(5) For the second set of the testing samples, when the volume percentage concentration of methane reaches $10 \%$, the practical concentrations of butane, ethylene, propylene, ethyne, carbon monoxide, and carbon dioxide are 0 . This test sample is just used to determine if the analysis results of other analytes suffer from the high concentration of methane. In fact, when the concentration of methane increases to such high concentration due to coal spontaneous combustion, the concentrations of other components such as $\mathrm{C}_{2} \mathrm{H}_{4}$ normally increase to several tens and even several hundreds of ppm [1]. On the other hand, in some cases such as coal bed methane outburst, both concentration of alkene and that of alkyne are very low [22]. At least, this is the case in China. Since the analysis results of alkene and alkyne do not suffer from alkane hydrocarbon when FTIRS is used to analyze the coal mine gases, the analysis results of FTIRS can also be used to distinguish the hazard of coal spontaneous combustion from that of coal bed methane outburst. In other words, the analysis results of FTIRS can also be used to forecast coal bed methane outburst directly.

\section{Conclusion}

In this paper, FTIRS has been used to analyze the gas mixture of coal spontaneous combustion. In particular, both the instrument parameters and analysis approach have been introduced. From the testing results and analysis, the following conclusions can be drawn.

(1) The analysis approach introduced in this paper is suitable for analyzing index gases of coal spontaneous combustion. The testing results show that ten components of the analyte can be analyzed accurately when the spectral resolution is $1 \mathrm{~cm}^{-1}$, optical path is $10 \mathrm{~cm}$, and Norton-Beer-medium function is used as the apodization function. Both the concentration of $\mathrm{CH}_{4}$ and that of $\mathrm{CO}_{2}$ range from 0 to $20 \%$ while that of others ranges from 0 to $3000 \times 10^{-6}$ or $5000 \times$ $10^{-6}$. The detection limit of every component of the analyte is less than $2 \times 10^{-6}$. Notably, both the detection limit of $\mathrm{C}_{2} \mathrm{H}_{2}$ and that of $\mathrm{C}_{2} \mathrm{H}_{4}$ are less than $0.5 \times 10^{-6}$. Although the detection accuracies of some components exceed the performance requirements in China when their concentrations are higher than $50 \times 10^{-6}$, this does not prohibit the usage of the FTIRS in the field of index gases monitoring because the effects of the analysis error of index gases brought to forecast result of coal spontaneous combustion are much less than that of spontaneous combustion experiment results of coal samples. The performance of the instrument developed in this work meets the technique requirements for analyzing the index gases of coal spontaneous combustion. In fact, detection limit can be decreased greatly if an optical path greater than $10 \mathrm{~cm}$ is used, since the sensitivity of gas depends on optical path length. Furthermore, it is not a problem if a lower detection limit is needed.

(2) Gas analysis instruments developed with FTIRS may become the preferred approach for monitoring coal safety because of its advantages. Firstly, because FTIRS does not produce exhausted gas, it is not a fire risk and is therefore a type of intrinsically safe instrumentation. Secondly, it does not need carrier gas and can be working for long time without maintenance. Also, it can potentially be used to monitor the index gases of coal spontaneous combustion in situ. Finally, it is possible to forecast coal bed methane outburst using this instrument since the concentrations of some index gases differ from each other.

(3) Although the developed instrument in this paper meets the technique requirements of index gases analysis of coal spontaneous combustion, there are still some measures that must be taken if FTIRS is needed to be used directly in coal mine to detect index gases of coal spontaneous combustion. At first, air conditioner is not permitted to be used in coal mine. Secondly, air pressure is not the same in different coal mines. Thus, at least, temperature and air pressure in coal mine must be monitored to compensate the error brought from the change of temperature and that of air pressure.

\section{Conflict of Interests}

The authors declare that there is no conflict of interests regarding the publication of this paper.

\section{Acknowledgments}

Authors are grateful for financial support by National Great Program on Science Instrument Development of China (2012YQ240127) and by National Natural Science Foundation of China (51277144).

\section{References}

[1] A. Adamus, J. Šancer, P. Guřanová, and V. Zubiček, "An investigation of the factors associated with interpretation of mine atmosphere for spontaneous combustion in coal mines," Fuel Processing Technology, vol. 92, no. 3, pp. 663-670, 2011. 
[2] R. V. K. Singh, "Spontaneous heating and fire in coal mines," Procedia Engineering, vol. 62, pp. 78-90, 2013.

[3] J. Xie, S. Xue, W. Cheng, and G. Wang, "Early detection of spontaneous combustion of coal in underground coal mines with development of an ethylene enriching system," International Journal of Coal Geology, vol. 85, no. 1, pp. 123-127, 2011.

[4] P. Lu, G. X. Liao, J. H. Sun, and P. D. Li, "Experimental research on index gas of the coal spontaneous at low-temperature stage," Journal of Loss Prevention in the Process Industries, vol. 17, no. 3, pp. 243-247, 2004.

[5] Apendix no. 5, Early Detection of Endogenous Fires, Regulation of the Minister of economy about health and safety at work, management of special fire protection in underground mines. Dz.U . no. 139, 1169, June 2002.

[6] Rozhodnutí č. 10/1990 obvodního báňského úřadu v Ostravě, č.j, Decision no. 10/1990 of the District Mining Office in Ostrava (OBÚ), ref. no. 10/1990-400-Ing.P/M1, OBÚ Ostrava, Ostrava, 1990.

[7] "The method of gas chromatography analysis and index optimization for mark gases of spontaneous combustion of coals stratum," AQ/T 1019, 2006. (Chinese).

[8] Y. L. Zhang, J. M. Wu, L. Chang, J. Wang, S. Xue, and Z. Li, "Kinetic and thermodynamic studies on the mechanism of lowtemperature oxidation of coal: A case study of Shendong coal (China)," International Journal of Coal Geology, vol. 120, pp. 4149, 2013.

[9] Q. W. Deng, X. H. Liu, C. Lu, Q. Z. Lin, and M. G. Yu, "Numerical simulation of spontaneous oxidation zone distribution in goaf under gas stereo drainage," Procedia Engineering, vol. 52, pp. 72-78, 2013.

[10] H.-Q. Zhu, Z.-Y. Song, B. Tan, and Y.-Z. Hao, "Numerical investigation and theoretical prediction of self-ignition characteristics of coarse coal stockpiles," Journal of Loss Prevention in the Process Industries, vol. 26, no. 1, pp. 236-244, 2013.

[11] V. Hanyecz, Á. Mohácsi, A. Pogány et al., "Multi-component photoacoustic gas analyzer for industrial applications," Vibrational Spectroscopy, vol. 52, no. 1, pp. 63-68, 2010.

[12] A. Sánchez, E. Eddings, and F. Mondragón, "Fourier transform infrared (FTIR) online monitoring of $\mathrm{NO}, \mathrm{N}_{2} \mathrm{O}$, and $\mathrm{CO}_{2}$ during oxygen-enriched combustion of carbonaceous materials," Energy and Fuels, vol. 24, no. 9, pp. 4849-4853, 2010.

[13] P. R. Griffiths and J. A. de Haseth, Fourier Transform Infrared Spectrometery, John Wiley \& Sons, 2nd edition, 2007.

[14] L. C. Speitel, "Fourier transform infrared analysis of combustion gases," Journal of Fire Sciences, vol. 20, no. 5, pp. 349-371, 2002.

[15] X. J. Tang, H. M. Hao, Y. J. Li, and J. H. Liu, "Analysis of mixed alkane gas based on Tikhonov regularization spectra selection and optimal neural network parameters selection," Spectroscopy and Spectral Analysis, vol. 31, no. 6, pp. 1673-1677, 2011.

[16] M. N. Leger and A. G. Ryder, "Comparison of derivative preprocessing and automated polynomial baseline correction method for classification and quantification of narcotics in solid mixtures," Applied Spectroscopy, vol. 60, no. 2, pp. 182-193, 2006.

[17] Y.-H. Fang, C. Kong, T.-G. Lan, W. Xiong, D.-M. Dong, and D.-C. Li, "Denoising and baseline correction of spectrum by wavelet transform," Optics and Precision Engineering, vol. 14, no. 6, pp. 1088-1092, 2006.

[18] A. F. Ruckstuhl, M. P. Jacobson, R. W. Field, and J. A. Dodd, "Baseline subtraction using robust local regression estimation," Journal of Quantitative Spectroscopy and Radiative Transfer, vol. 68, no. 2, pp. 179-193, 2001.
[19] Z.-M. Zhang, S. Chen, and Y.-Z. Liang, "Baseline correction using adaptive iteratively reweighted penalized least squares," Analyst, vol. 135, no. 5, pp. 1138-1146, 2010.

[20] J. Zhao, H. Lui, D. I. Mclean, and H. Zeng, "Automated autofluorescence background subtraction algorithm for biomedical raman spectroscopy," Applied Spectroscopy, vol. 61, no. 11, pp. 1225-1232, 2007.

[21] F. Stout, J. H. Kalivas, and K. Héberger, "Wavelength selection for multivariate calibration using tikhonov regularization," Applied Spectroscopy, vol. 61, no. 1, pp. 85-95, 2007.

[22] F. H. An and Y. P. Cheng, "An explanation of large-scale coal and gas outbursts in underground coal mines: the effect of low-permeability zones on abnormally abundant gas," Natural Hazards and Earth System Science, vol. 1, pp. 4751-4775, 2013. 

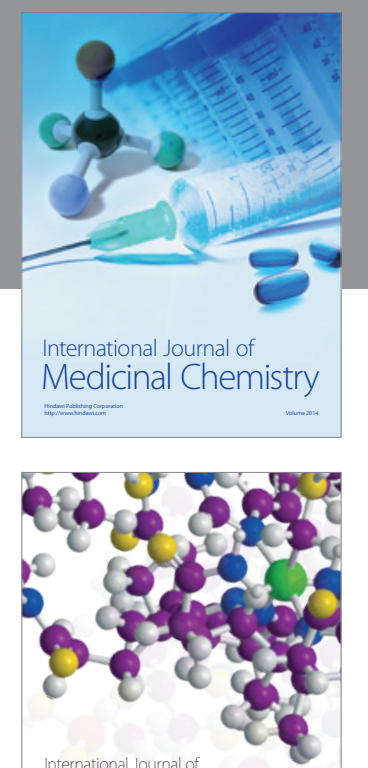

\section{Carbohydrate} Chemistry

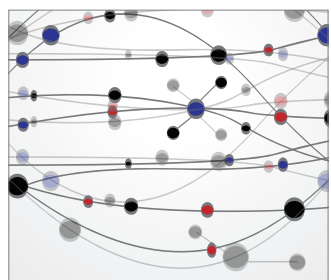

The Scientific World Journal
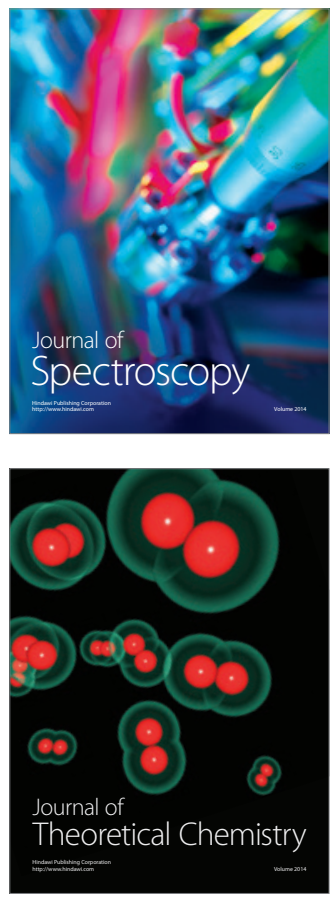
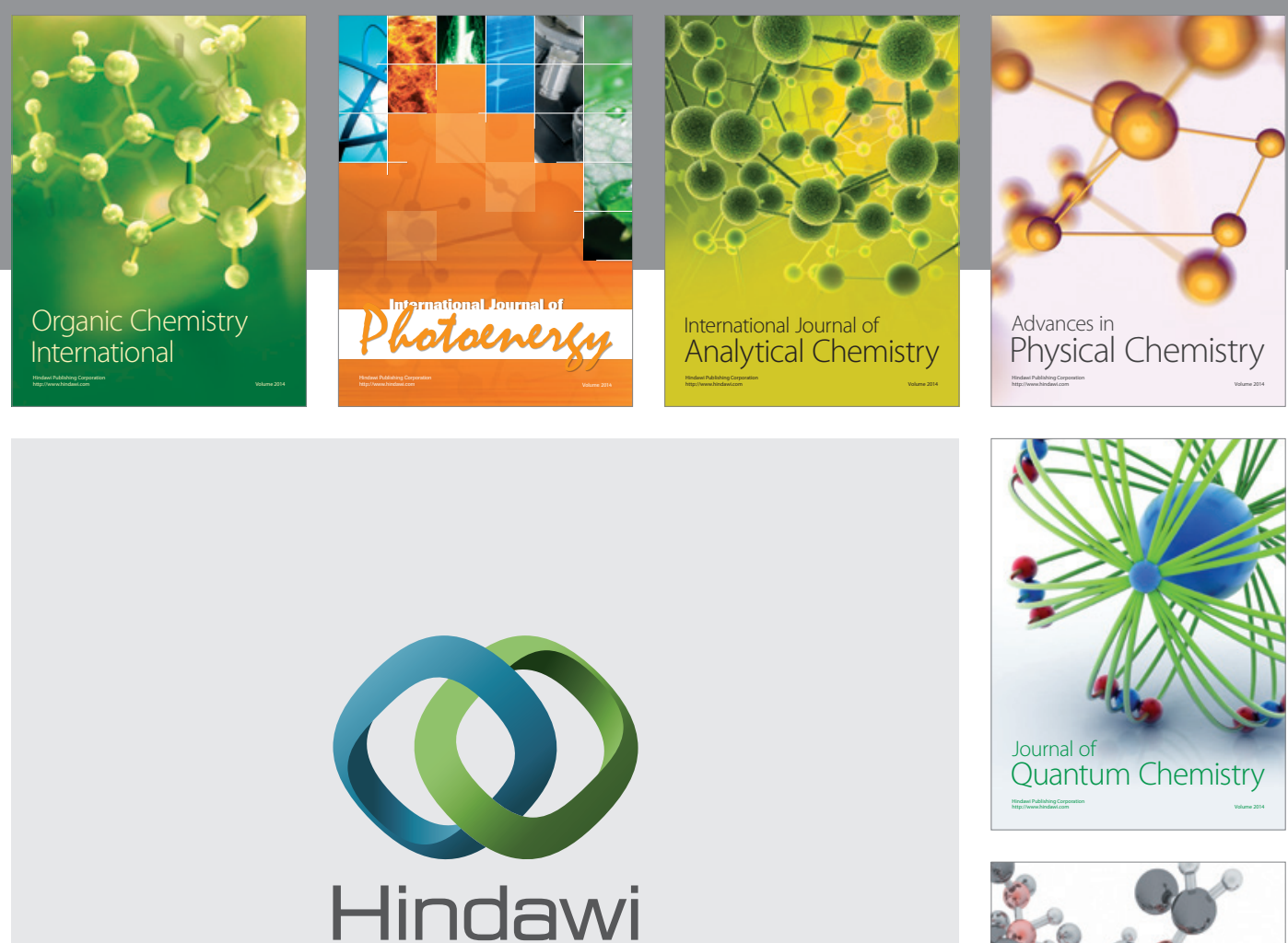

Submit your manuscripts at

http://www.hindawi.com

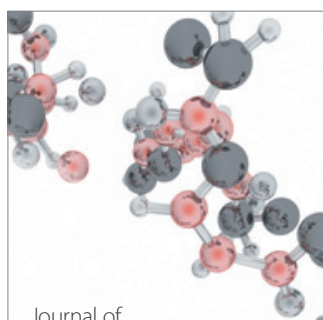

Analytical Methods

in Chemistry

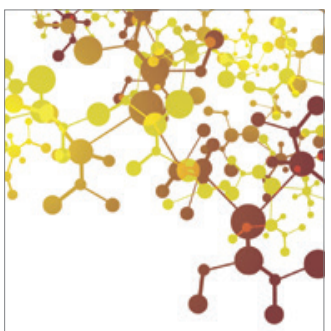

Journal of

Applied Chemistry

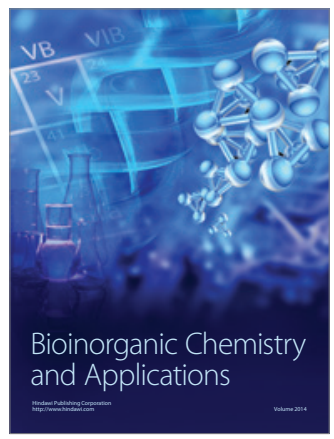

Inorganic Chemistry
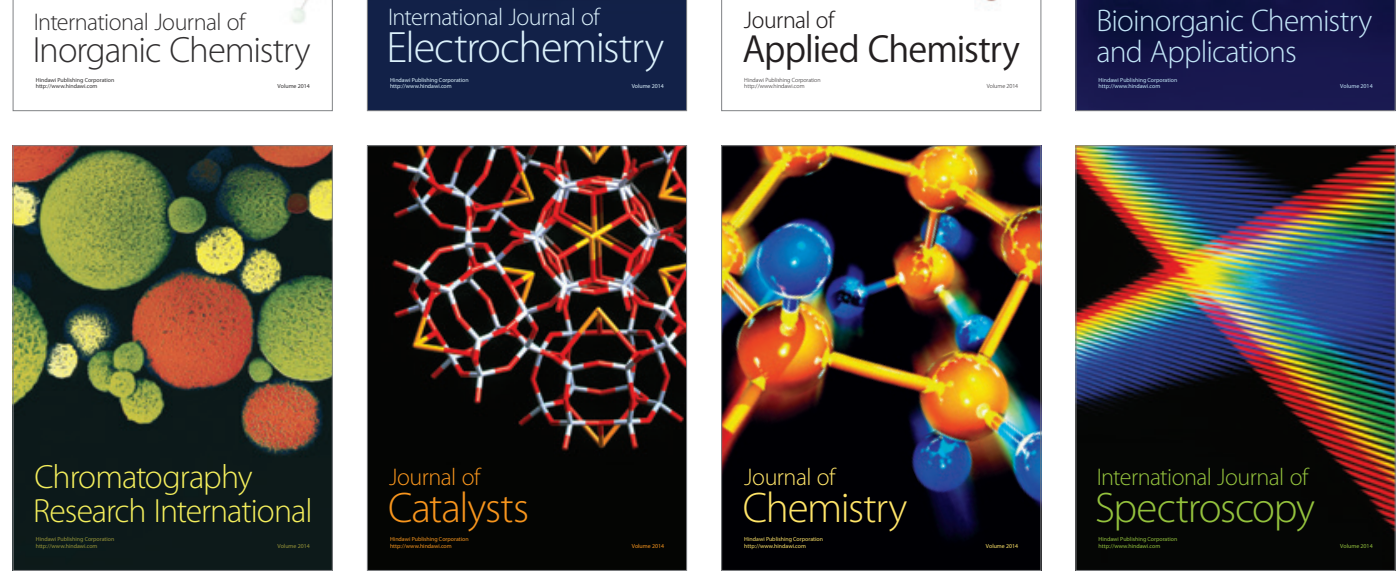